\title{
TAK-242 exerts a neuroprotective effect via suppression of the TLR4/MyD88/TRIF/NF-кB signaling pathway in a neonatal hypoxic-ischemic encephalopathy rat model
}

\author{
LIJUN JIANG $^{1,2^{*}}$, ZHENXING XU $^{1 *}$, HUI LI $^{3 *}$, MINGFU WU ${ }^{1}$, FUDONG WANG ${ }^{1}$, \\ SHUNYING LIU ${ }^{1}$, JIANLAN TAO ${ }^{1}$ and XING FENG ${ }^{2}$
}

\author{
${ }^{1}$ Department of Neonatology, Affiliated Hospital of Yangzhou University, Yangzhou, Jiangsu 225000; \\ ${ }^{2}$ Department of Neonatology, Affiliated Children's Hospital of Soochow University, Suzhou, Jiangsu 215000; \\ ${ }^{3}$ Department of Interventional Medicine, Yangzhou Hongquan Hospital, Yangzhou, Jiangsu 225200, P.R. China
}

Received August 14, 2019; Accepted April 6, 2020

DOI: $10.3892 / \mathrm{mmr} .2020 .11220$

\begin{abstract}
Neonatal hypoxic-ischemic encephalopathy (HIE) is one of the main causes of death and nervous system damage in neonates. The aim of the present study was to investigate the effect of the Toll-like receptor 4 (TLR4) antagonist TAK-242 on HIE. The Rice-Vannucci method was used for ligation of the left common carotid artery, followed by hypoxic treatment for $2.5 \mathrm{~h}$ to establish a neonatal HIE rat model. Rats were intraperitoneally injected with $7.5 \mathrm{ml} / \mathrm{kg}$ TAK-242 after hypoxia-ischemia. It was demonstrated that TAK-242 significantly reduced the infarct volume and cerebral edema content of neonatal rats after HIE, alleviating neuronal damage and neurobehavioral function deficits. Furthermore, TAK-242 decreased the protein expression levels of TLR4, MyD88, TIR-domain-containing adapter-inducing interferon- $\beta$ (TRIF), NF- $\kappa B$, tumor necrosis factor $\alpha$ (TNF- $\alpha$ ) and interleukin- $1 \beta$ in the hippocampus. The present results suggested that TAK-242 may exert a neuroprotective effect after HIE by inhibiting the TLR4/MyD88/TRIF/NF- $\mathrm{kB}$ signaling pathway, and reducing the release of downstream inflammatory cytokines.
\end{abstract}

\section{Introduction}

Neonatal hypoxic-ischemic encephalopathy (HIE) is a type of brain damage that occurs as a result of partial or complete

Correspondence to: Dr Xing Feng, Department of Neonatology, Affiliated Children's Hospital of Soochow University, 92 Nanbert Zhong Street, Suzhou, Jiangsu 215000, P.R. China

E-mail: xingfengsoochow@163.com

${ }^{*}$ Contributed equally

Abbreviations: HIE, hypoxic-ischemic encephalopathy; TLRs, toll-like receptors; IRFs, interferon regulatory factors; OD, optical density

Key words: TAK-242, HIE, TLR4, NF-кB hypoxia of brain tissues, which decreases or suspends the cerebral blood flow in neonates (1). HIE is one of the main reasons for neonatal death, secondary mental retardation, cerebral palsy and other disabilities (2). It is estimated that neonatal HIE accounts for $25 \%$ of global neonatal deaths annually (1). Brain damage due to HIE is the predominant cause of mortality in neonates, and the proportion is as high as $14.5 \%$ in pediatric patients with cerebral palsy caused by HIE, resulting in a substantial burden on pediatrics, families and society (3-6). Previous studies have demonstrated that the cascade reaction of proinflammatory cytokines in brain tissue is activated after hypoxia-ischemia condition, which in turn activates inflammatory factors and further aggravates brain damage (7-9). Therefore, secondary brain injury plays an important role in the pathogenesis of HIE, and inhibition of the inflammatory response may protect hypoxic-ischemic brain tissues $(1,10)$.

Toll-like receptors (TLRs) are crucial in the induction and regulation of the inflammatory response (11). TLR4 activates downstream protein kinases via MyD88-dependent and TIR-domain-containing adapter-inducing interferon- $\beta$ (TRIF)-dependent signaling pathways, subsequently leading to the activation of NF- $\kappa \mathrm{B}$ and interferon regulatory factors (IRFs) (11). This activation then induces and regulates the release of immune inflammatory factors (12). NF- $\kappa \mathrm{B}$ is a transcriptional regulator commonly found in mammalian cells, and specifically binds to sequence-tagged sites of promoters and enhancers of various cellular immune-related genes (13). By regulating the expression levels of various cytokines, NF- $\kappa \mathrm{B}$ plays an essential role in immune and inflammatory responses (13). The release of various immune inflammatory factors depends on the activation of the NF- $\kappa \mathrm{B}$ signaling pathway induced by TLR4, thus activating NF- $\kappa \mathrm{B}$ acts as the central link for TLR4 in regulating inflammatory responses (14).

The TLR4/NF- $\kappa \mathrm{B}$ signaling pathway plays a vital role in the activation of the inflammatory response and development of brain damage caused by cerebral ischemia-hypoxia, carbon monoxide poisoning and brain trauma (15-20). A TLR4 antagonist alleviates various brain injuries, such as ischemic stroke, subarachnoid hemorrhage and brain trauma (21-23). 
However, the role of the TLR4/NF- $\mathrm{BB}$ signaling pathway in neonatal HIE and its regulatory mechanisms are not fully understood. TAK-242 is a micromolecular TLR4-specific inhibitor that selectively binds to the intracellular domain of TLR4 to inhibit the production of inflammatory mediators (24). A previous study has demonstrated that TAK-242 crosses the blood-brain barrier, blocks the TLR4 signaling pathway, inhibits phosphorylation of downstream proteins and downregulates the expression level of inflammatory cytokines, thereby improving brain neural function and reducing cerebral ischemia-reperfusion injury in mice (25). However, the role of TAK-242 in the inhibition of the TLR4/NF- $\kappa$ B signaling pathway activation, the release of inflammatory cytokines and its effect as a neuroprotectant after HIE in neonatal rats requires further investigation.

In the present study, a neonatal HIE rat model was established to investigate the role of the TLR4/NF- $\kappa \mathrm{B}$ signaling pathway in the infarct volume, degree of cerebral edema, pathomorphological changes of brain tissue, neurobehavioral function deficits and signaling pathway-related protein levels after HIE. TAK-242 was intraperitoneally injected into rats $30 \mathrm{~min}$ after hypoxia-ischemia to investigate whether TAK-242 alleviates brain damage after HIE by inhibiting the TLR4/NF- $\kappa \mathrm{B}$ signaling pathway and reducing the release of inflammatory cytokines. The present results may help to facilitate the development of a new therapeutic method for HIE treatment in clinical practice.

\section{Materials and methods}

Laboratory animals. A total of 15 female Sprague-Dawley (SD) rats (age, 12 weeks; weight, 260-300 g) with a litter size of 10-12 neonatal rats were purchased from the Laboratory Animal Center of Yangzhou University [license no. SCXK (Su) 2017-0007]. Female rats were allowed free access to food and water. Neonatal SD rats (age, 7 days; weight, 12-18 g) were freely fed by female rats at $24 \pm 2^{\circ} \mathrm{C}$ with a relative humidity of $55 \pm 5 \%$ and an alternate $12 \mathrm{~h}$ light/dark cycle.

All animal experimental procedures were approved by the Laboratory Animal Ethics Committee of Yangzhou University, and were conducted in strict accordance with the Regulation on the Administration of Laboratory Animals issued by the Ministry of Science and Technology of the People's Republic of China. All efforts were made to reduce the suffering of the animals.

Animal grouping and animal models. A total of 114 SD rats (age, 7 days) were randomly assigned into the sham $(n=30)$, HIE + vehicle $(\mathrm{n}=42), \mathrm{HIE}+0.75 \mathrm{mg} / \mathrm{kg}$ TAK-242 $(\mathrm{n}=6)$, $\mathrm{HIE}+1.5 \mathrm{mg} / \mathrm{kg}$ TAK-242 ( $\mathrm{n}=6)$ or HIE $+3 \mathrm{mg} / \mathrm{kg}$ TAK-242 $(n=30)$ groups. The HIE model was established according to the Rice-Vannucci method (26). Anesthesia was induced by inhalation of 2-3\% isoflurane (Merck Hoei Ltd.) and maintained using 1-1.5\% isoflurane using an animal general anesthesia machine. After inhalation of anesthesia, neonatal rats were fixed on an operating table at a constant temperature in the supine position and disinfected with $75 \%$ alcohol on the skin of middle neck. The skin was cut along the median line $\sim 0.5 \mathrm{~cm}$. The subcutaneous fat and superficial fascia were incised layer by layer, the muscle and connective tissue were separated blunt by bending forceps, and the left common carotid artery was carefully separated. The 5-0 surgical suture was used to ligate both ends and the blood vessel was disconnected in the middle. Suturing was performed, and then the surgical incision was disinfected with $75 \%$ alcohol. After surgery, the neonatal rats were placed in an incubator at $37^{\circ} \mathrm{C}$ for recovery for $1 \mathrm{~h}$, and then placed in an anoxic box with a nitrogen-oxygen mixed gas $\left(8 \% \mathrm{O}_{2}+92 \% \mathrm{~N}_{2}\right)$ delivered at a flow rate of $21 / \mathrm{min}$ and a controlled temperature at $36 \pm 1^{\circ} \mathrm{C}$ for $2.5 \mathrm{~h}$. In the sham group, only the left common carotid artery was separated, and no ligation or hypoxic treatment were performed. Rats in HIE + TAK-242 group were intraperitoneally injected with $0.75,1.5$ and $3 \mathrm{mg} / \mathrm{kg}$ of TAK-242 isopyknic drug solution, for $30 \mathrm{~min}$ after hypoxia-ischemia. The HIE + vehicle group was injected with an equal volume $(7.5 \mathrm{ml} / \mathrm{kg})$ of vehicle (10\% DMSO). TAK-242 (InvivoGen) solution was prepared, and administered as previously described $(25,27,28)$.

\section{Behavioral experiments}

Neurological deficit score. In total, six neonatal rats in each group were randomly selected for neurological deficit score using the Longa scale $48 \mathrm{~h}$ after hypoxia-ischemia (29) as follows: i) 0 points, no significant neurological deficit, with normal mobility of limbs; ii) 1 point, mild neurological deficit, with an inability to fully extend the right forelimb when lifting the tails; iii) 2 points, moderate neurological deficit, with turning rightwards while walking; iv) 3 points, severe neurological deficit, with falling down rightwards while walking; and v) 4 points, inability to walk spontaneously and a loss of consciousness. This was assessed with rats walking along a table.

Rotarod task and beam walking test. In total, six neonatal rats in each group were randomly selected to undergo the rotarod task and beam walking test at week 4 after hypoxia-ischemia, in order to test motor integration and coordination abilities (19). The rats were trained for 3 days before undergoing the formal experiment. For the rotarod task, rats were placed on a rotating rod and the rotation speed was slowly increased from $4 \mathrm{rpm}$ to $40 \mathrm{rpm}$ within $5 \mathrm{~min}$. The time of maintaining balance to falling down from the rotating rod was recorded.

For the beam walking test, a balance beam with a length of $100 \mathrm{~cm}$ and a width of $2.0 \mathrm{~cm}$ was placed at a height of $\sim 50 \mathrm{~cm}$ from the ground. A black box was placed at one end of the balance beam, and the time taken for the rats to cross the balance beam to reach the black box was recorded.

Determination of infarct volume. Then, six neonatal rats in each group were randomly selected for triphenyltetrazolium chloride (TTC) staining after $48 \mathrm{~h}$ of hypoxia-ischemia to determine the infarct volume. Neonatal rats were deeply anesthetized with $3 \%$ isoflurane and sacrificed by cervical dislocation. After the heartbeat, respiratory arrest and reflex disappeared, the brains were dissected and coronally sectioned to $\sim 2 \mathrm{~mm}$ slices. The brain sections were placed in $2 \%$ 2,3,5-TTC solution (Thermo Fisher Scientific, Inc.), incubated for $30 \mathrm{~min}$ in dark place at $37^{\circ} \mathrm{C}$ and then immersed in $10 \%$ formaldehyde solution for fixation at $4^{\circ} \mathrm{C}$ overnight. ImageJ software (version 1.8.0, National Institutes of Health) was used to trace and analyze infarct volume (30). 
Determination of brain water content. In total, six neonatal rats in each group were randomly selected after $48 \mathrm{~h}$ of hypoxia-ischemia to calculate the brain water content in order to assess the degree of cerebral edema. Neonatal rats were deeply anesthetized, sacrificed by cervical dislocation and the brains were removed after the heartbeat, respiratory arrest and reflex disappeared. The water and blood stains on the brain surface were removed using filter paper to weigh the wet weight of the brain (31). The brain tissues were wrapped in a tin foil paper and dried at $100^{\circ} \mathrm{C}$ in an oven for $12 \mathrm{~h}$ until a constant weight was reached, and then were measured at room temperature to weigh the dry weight of the brain. The brain water content was calculated by dry-wet specific gravity method as follows: Brain water content=(brain wet weightbrain dry weight)/brain wet weight x $100 \%$ (31).

Hematoxylin and eosin (HE) staining, and immunohistochemistry. Neonatal rats $(\mathrm{n}=6)$ were randomly selected from each group to undergo heart perfusion with PBS, and were fixed with $4 \%$ paraformaldehyde under deep anesthesia after $48 \mathrm{~h}$ of hypoxia-ischemia. HE staining and immunohistochemical analysis of $5-\mu \mathrm{m}$ brain tissue sections were performed as previously described $(17,19)$. Pathological changes were then observed under a light microscope (magnification, x400; Nikon Corporation) after HE staining. Paraffin sections were dewaxed and endogenous peroxidase activity was blocked with $3 \% \mathrm{H}_{2} \mathrm{O}_{2}$ solution at room temperature for $5 \mathrm{~min}$, and $5 \%$ goat serum (cat. no. C0265; Beyotime Biotech Co., Ltd.) was used for mounting. The following primary antibodies were added: Rabbit polyclonal anti-TLR4 (1:100; cat. no. 19811-1-AP; ProteinTech Group,Inc.) and rabbit polyclonal anti-phosphorylated (p)-NF- $\mathrm{B}$ p65 (1:100; cat. no. AP0123; ABclonal Biotech Co., Ltd.), which were then incubated overnight at $4^{\circ} \mathrm{C}$ and rewarmed at room temperature. The anti-rabbit biotin-conjugated IgG secondary antibody (1:100; cat. no. P0628; Beyotime Biotech Co., Ltd.) were added, incubated at $37^{\circ} \mathrm{C}$ for $1 \mathrm{~h}$ and horseradish peroxidase was added for labeling for $15 \mathrm{~min}$ at room temperature. Color development was performed using 3,3'-diaminobenzidine, and hematoxylin was used for counterstaining at room temperature. Immunohistochemical images were then observed under a light microscope (magnification, x400; Nikon Corporation). Analysis was performed using ImageJ software.

Western blotting. Neonatal rats $(n=6)$ in each group were randomly selected to undergo heart perfusion with PBS under deep anesthesia at 24, 48 and $72 \mathrm{~h}$ after hypoxia-ischemia, and then the left hippocampus was removed. The tissues were homogenized with RIPA lysis buffer (cat. no. P0013K; Beyotime Biotech Co., Ltd.). The samples were incubated on ice for $30 \mathrm{~min}$, and the supernatants were recovered by centrifugation at $13,400 \mathrm{x}$ g at $4^{\circ} \mathrm{C}$ for $20 \mathrm{~min}$. Protein concentrations were determined by bicinchoninic acid protein assay, and the samples were incubated with $5 \mathrm{x}$ Loading buffer (cat. no. P0015; Beyotime Biotech Co., Ltd.) at $100^{\circ} \mathrm{C}$ for $5 \mathrm{~min}$. Western blotting was performed as described previously (19). In total, $50 \mu \mathrm{g}$ of protein was taken from each sample and separated by $10 \%$ SDS-PAGE, and then transferred onto a PVDF membrane (EMD Millipore). Then, $5 \%$ skim milk powder was used for blocking at room temperature for $2 \mathrm{~h}$, and the following primary antibodies, rabbit polyclonal anti-TLR4 (1:500; cat. no. 19811-1-AP; ProteinTech Group, Inc.), rabbit polyclonal anti-MyD88 (1:1,000; cat. no. A0980; ABclonal Biotech Co., Ltd.), rabbit polyclonal anti-TRIF (1:500; cat. no. ab13810; Abcam), rabbit polyclonal anti-phospho-NF-кB p65 (1:1,000; cat. no. AP0123; ABclonal Biotech Co., Ltd.) and rabbit polyclonal anti-NF-kB (1:1,000; cat. no. A2547; ABclonal Biotech Co., Ltd.) were added and incubated overnight at $4^{\circ} \mathrm{C}$. The anti-rabbit HRP-conjugated IgG secondary antibody (1:5,000; cat. no. AS014; ABclonal Biotech Co., Ltd.) was incubated for $2 \mathrm{~h}$ at room temperature, developed and visualized by Hypersensitive ECL chemiluminescence Kit (cat. no. P0018AS; Beyotime Institute of Biotechnology) in a dark room. Densitometry of protein bands was performed using ImageJ software.

ELISA. The supernatant of the left hippocampus was collected after homogenization and centrifugation as described above. The levels of the inflammatory cytokines tumor necrosis factor $\alpha(\mathrm{TNF}-\alpha)$ and interleukin (IL)- $1 \beta$ in each group were determined using TNF- $\alpha$ and IL-1 $\beta$ ELISA kits (cat. nos. RK00029 and RK00009; ABclonal Biotech Co., Ltd.) according to the manufacturer's instructions. The absorbance was detected at $450 \mathrm{~nm}$ and the content of each sample was calculated from the standard curve.

Statistical analysis. Statistical analyses were performed using SPSS 19.0 software (SPSS, Inc.). Normally distributed data are presented as the mean $\pm \mathrm{SD}$, and comparisons among groups was performed using a one-way ANOVA followed by Tukey's test. Non-normally distributed data are presented as the median (interquartile range). A Mann-Whitney $U$ test was used for the statistical analysis of neurological deficit score, and the Dunn-Bonferroni test was used for further comparison between the groups. All experiments were repeated three times. $\mathrm{P}<0.05$ was considered to indicate a statistically significant difference.

\section{Results}

Activation of the TLR4/MyD88/TRIF/NF- $\mathrm{KB}$ signaling pathway and release of inflammatory cytokines during brain damage after HIE. The protein expression levels of TLR4, MyD88, TRIF, p-NF- $\kappa$ B, TNF- $\alpha$ and IL- $1 \beta$ in the left hippocampus of neonatal rats were significantly increased after HIE. These protein expression levels reached a peak at $48 \mathrm{~h}$, and started to decrease at $72 \mathrm{~h}$ after HIE (Fig. 1).

TAK-242 alleviates neurological deficits and improves neurobehavioral function after HIE. It was observed that the right forelimb of neonatal rats in the HIE + vehicle group did not extend when tailing and the rats turned rightwards while walking, four rats also fell down towards the right side. Furthermore, rats in the HIE + vehicle group could not walk spontaneously, and thus exhibited significant signs of neurological deficit. All three doses of TAK-242 alleviated neurological deficit after HIE, and the high dose group ( $3 \mathrm{mg} / \mathrm{kg}$ ) was identified to be the most effective compared with the vehicle group (Fig. 2A).

The neurobehavioral function test results in the HIE + vehicle group were significantly lower compared with the sham group. 

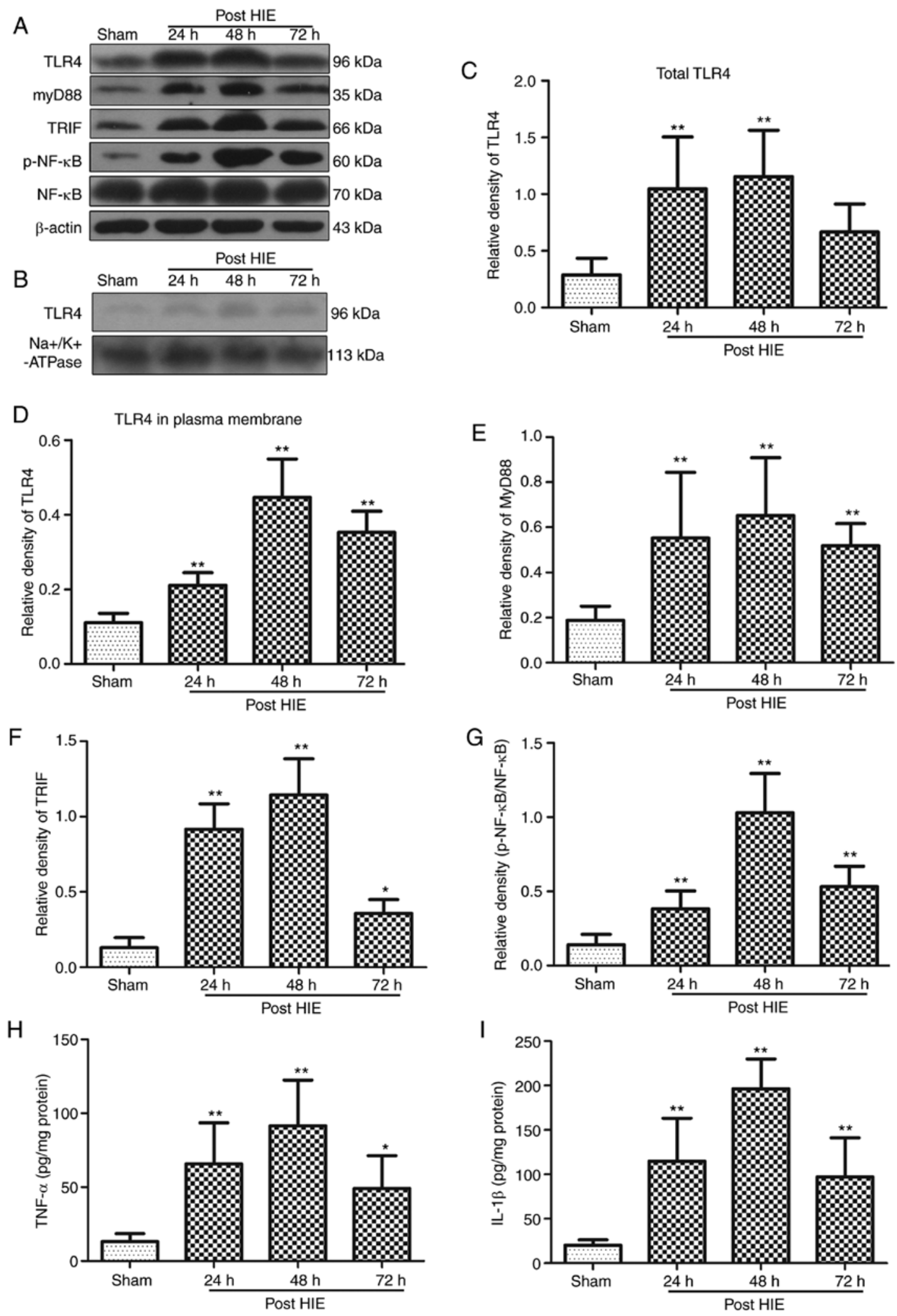

Figure 1. Activation of the TLR4/MyD88/TRIF/NF-kB signaling pathway and release of inflammatory cytokines during brain damage after HIE. (A) Representative

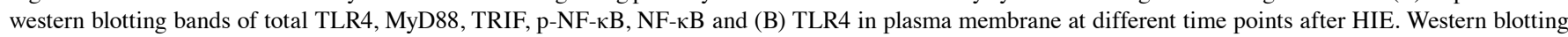
and ELISA results indicated that the levels of (C) total TLR4, (D) TLR4 in plasma membrane, (E) MyD88, (F) TRIF, (G) p-NF-kB, (H) TNF- $\alpha$ and (I) IL-1 $\beta$ were significantly increased and peaked at $48 \mathrm{~h}$, but were gradually decreased at $72 \mathrm{~h} . \mathrm{n}=6$ per group ${ }^{*} \mathrm{P}<0.05,{ }^{* *} \mathrm{P}<0.01$ vs. sham group. $\mathrm{P}$-, phosphorylated; TNF- $\alpha$, tumor necrosis factor $\alpha$; IL, interleukin; TLR4, Toll like receptor 4; TRIF, TIR-domain-containing adapter-inducing interferon- $\beta$; HIE, hypoxic-ischemic encephalopathy,

Moreover, compared with the HIE + vehicle group, the rotarod running time exhibited a significant increase in the HIE + TAK-242 (3 mg/kg) group, while beam walking latency was significantly reduced, thus demonstrating improved motor integration and coordination abilities after TAK-242 treatment (Fig. 2B and C). 

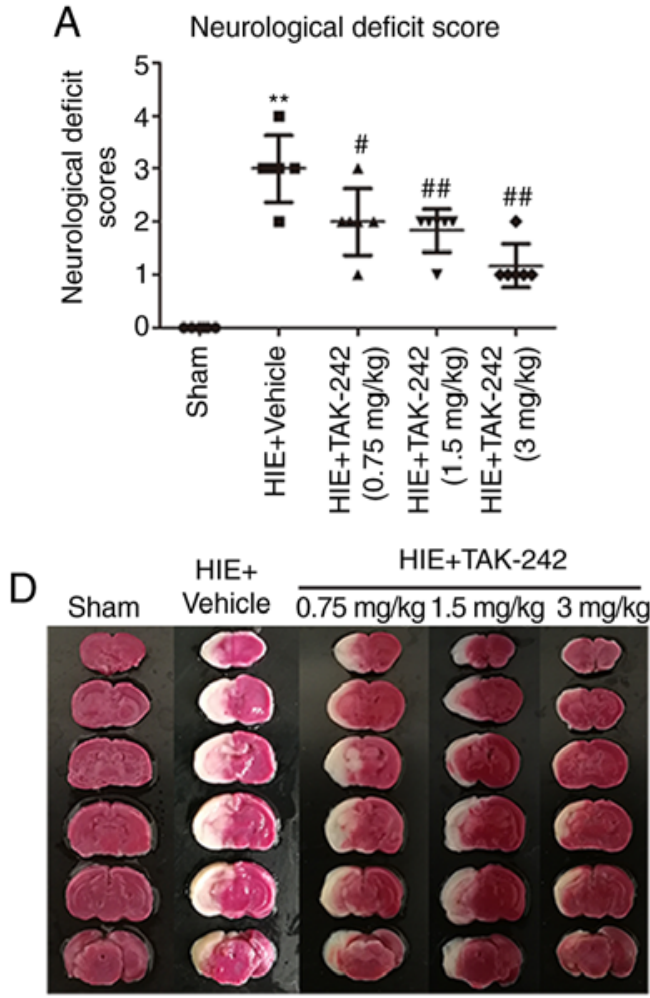
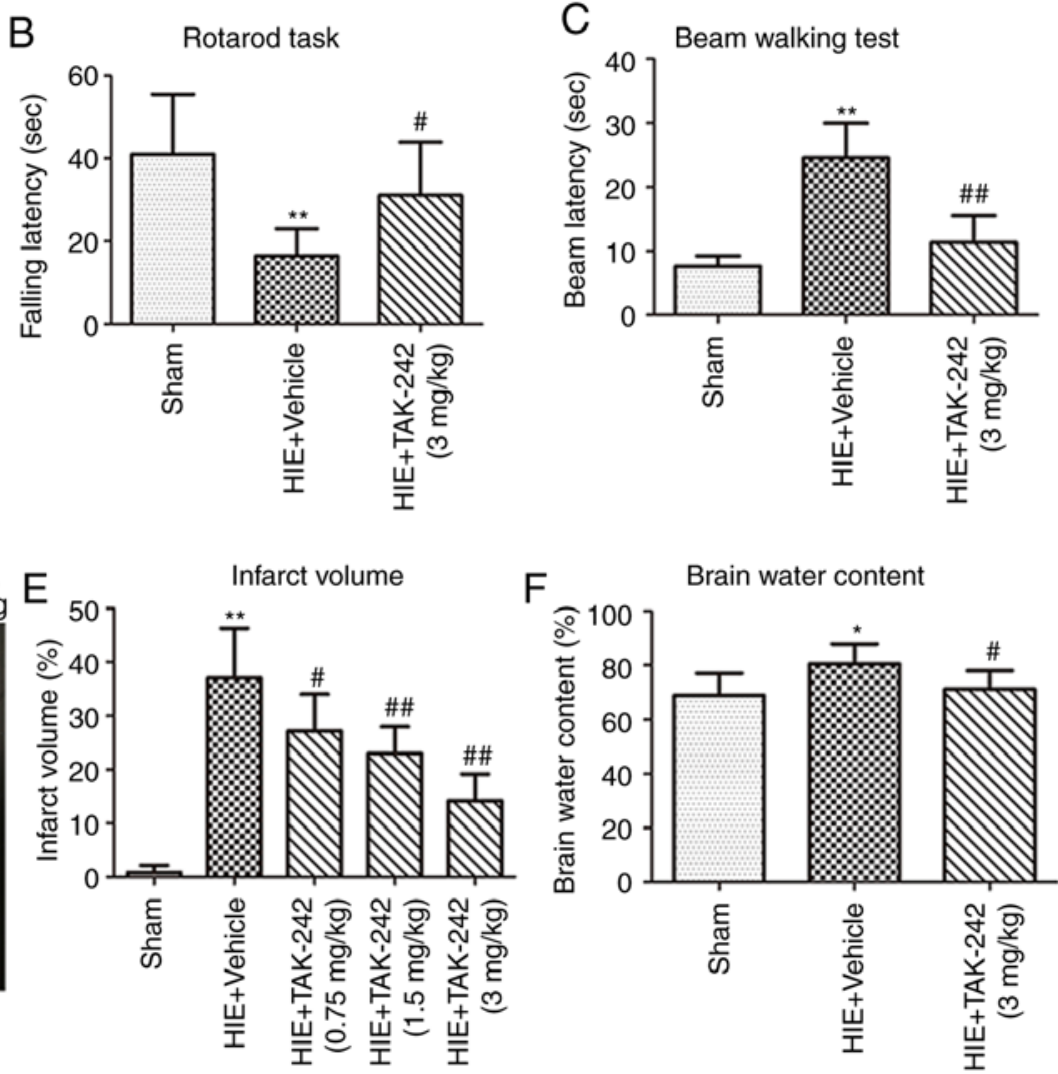

Figure 2. TAK-242 alleviates neurological deficits, improves neurobehavioral function, and reduces infarct volume and cerebral edema content after HIE. Low, medium and high doses $(0.75,1.5$ and $3 \mathrm{mg} / \mathrm{kg})$ of TAK-242 alleviated neurological deficits in neonatal rats $48 \mathrm{~h}$ after HIE, with high dose group ( $3 \mathrm{mg} / \mathrm{kg})$ appearing the most effective. (A) TAK-242 (3 mg/kg) significantly improved the (B) rotarod task and (C) beam walking test scores in neonatal rats 4 weeks after HIE. (D) Triphenyltetrazolium chloride staining of representative brain coronal sections in each group. (E) Low, medium and high doses of TAK-242 significantly reduced the infarct volume of neonatal rats after HIE, with high dose group demonstrating the most significant decrease. (F) TAK-242 (3 mg/kg) significantly reduced the degree of brain edema in neonatal rats after HIE. $\mathrm{n}=6$ per group. ${ }^{*} \mathrm{P}<0.05,{ }^{* *} \mathrm{P}<0.01$ vs. sham group; ${ }^{\#} \mathrm{P}<0.05$, ${ }^{\# \#} \mathrm{P}<0.01$ vs. HIE + vehicle group. HIE, hypoxic-ischemic encephalopathy.

TAK-242 reduces infarct volume and the content of cerebral edema $48 \mathrm{~h}$ after HIE. A large-area infarct was observed in the left cerebral hemisphere of the neonatal rats in the HIE + vehicle group, while the infarct volume of the neonatal rats in the three TAK-242 groups was significantly reduced compared with the HIE + vehicle group, with the high dose having the greatest significant difference (Fig. 2D and E). Furthermore, the present results suggested that the brain edema content of neonatal rats in the TAK- 242 group $(3 \mathrm{mg} / \mathrm{kg}$ ) was significantly decreased compared with the HIE + vehicle group (Fig. 2F).

TAK-242 alleviates pathological damage of brain tissue after HIE. It was demonstrated that the structure of left hippocampus in the sham group was ordered, and the neuronal cells were aligned and compact. Moreover, the cell morphology was normal. In the HIE + vehicle group, the structure of the hippocampus was disordered, and the neuronal cells were loosely arranged and irregular. Furthermore, cellular edema, karyopyknosis, karyorrhexis and necrosis of nerve cells were observed. Moreover, it was demonstrated that in the HIE + TAK-242 group (3 mg/kg), hippocampal damage was reduced compared with the HIE + vehicle group (Fig. 3A).

TAK-242 inhibits protein expression of the TLR4/MyD88/

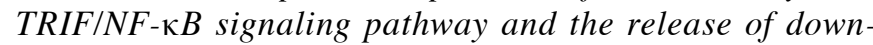

stream inflammatory cytokines in the hippocampus. To further investigate the neuroprotective mechanism of TAK-242 in HIE, immunohistochemistry was performed to determine the expression levels of TLR4 and $\mathrm{p}-\mathrm{NF}-\kappa \mathrm{B}$ in the left hippocampus. It was demonstrated that the expression levels of TLR4 and p-NF- $\kappa \mathrm{B}$-positive cells in the hippocampus were significantly increased after $48 \mathrm{~h}$ of HIE, but were significantly decreased after treatment with $3 \mathrm{mg} / \mathrm{kg}$ TAK-242 (Fig. 3B-E). Furthermore, western blotting and ELISA results suggested that the levels of TLR4, MyD88, TRIF, p-NF- $\kappa$ B, TNF- $\alpha$ and IL-1 $\beta$ in the left hippocampus in the HIE + vehicle group peaked after $48 \mathrm{~h}$ of HIE. Moreover, the present results suggested that administration of $3 \mathrm{mg} / \mathrm{kg}$ TAK-242 significantly decreased the expression levels of TLR4, MyD88, TRIF, p-NF- $\kappa \mathrm{B}, \mathrm{TNF}-\alpha$ and IL- $1 \beta$ in the hippocampus after $48 \mathrm{~h}$ of HIE, compared with the vehicle group (Fig. 4).

\section{Discussion}

In the present study, results from the neonatal HIE rat model suggested that TAK-242 treatment had significantly reduced the infarct volume and cerebral edema content. Furthermore, TAK-242 alleviated neuronal damage, improved neurobehavioral function and decreased the protein expression levels of TLR4, MyD88, TRIF, $p-N F-\kappa B$, TNF- $\alpha$ and IL- $1 \beta$ in the hippocampus. 
A
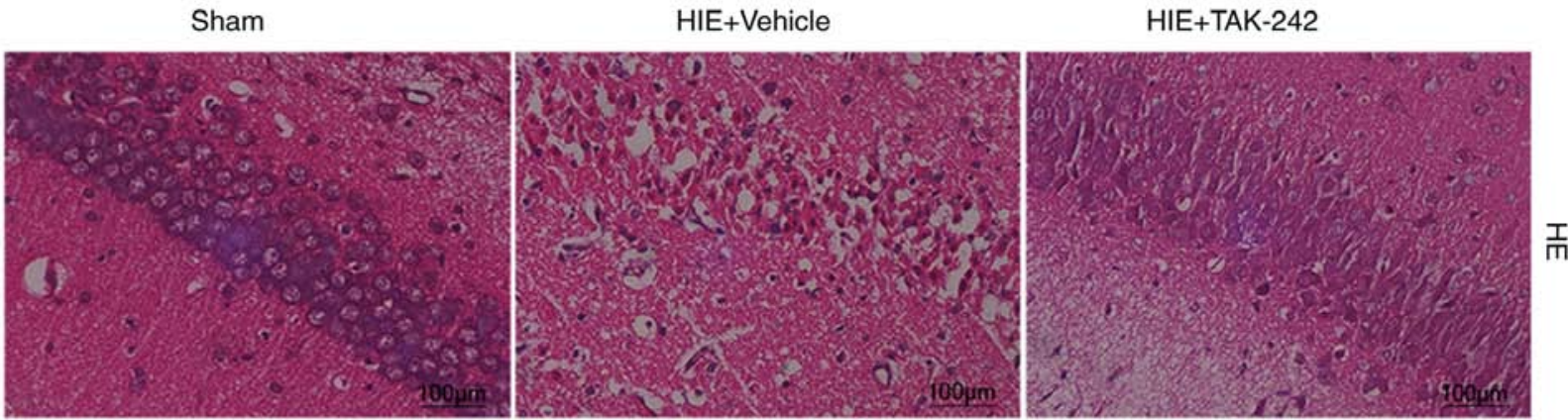

B
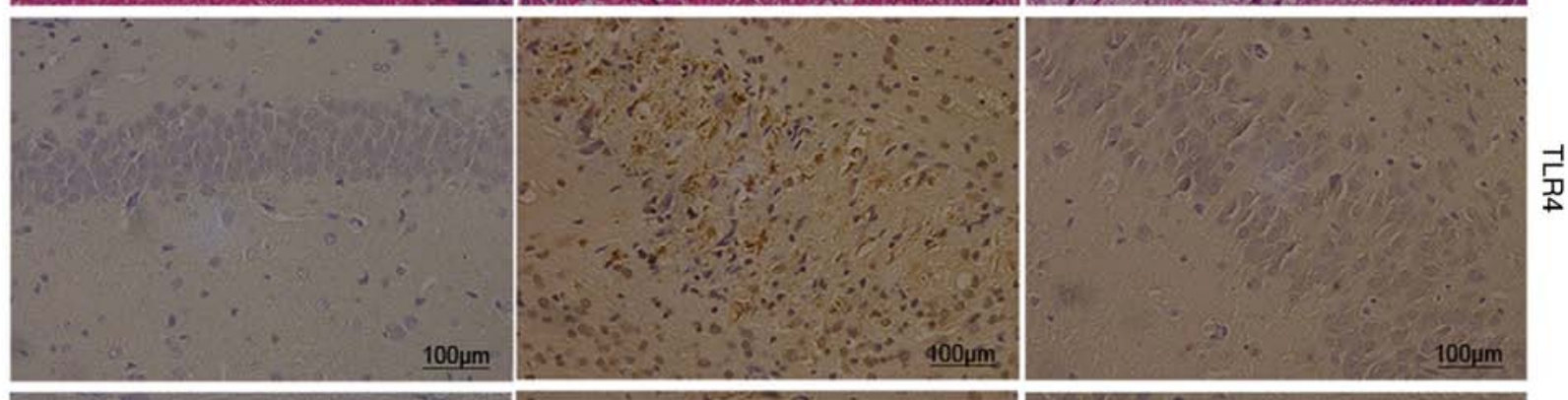

C
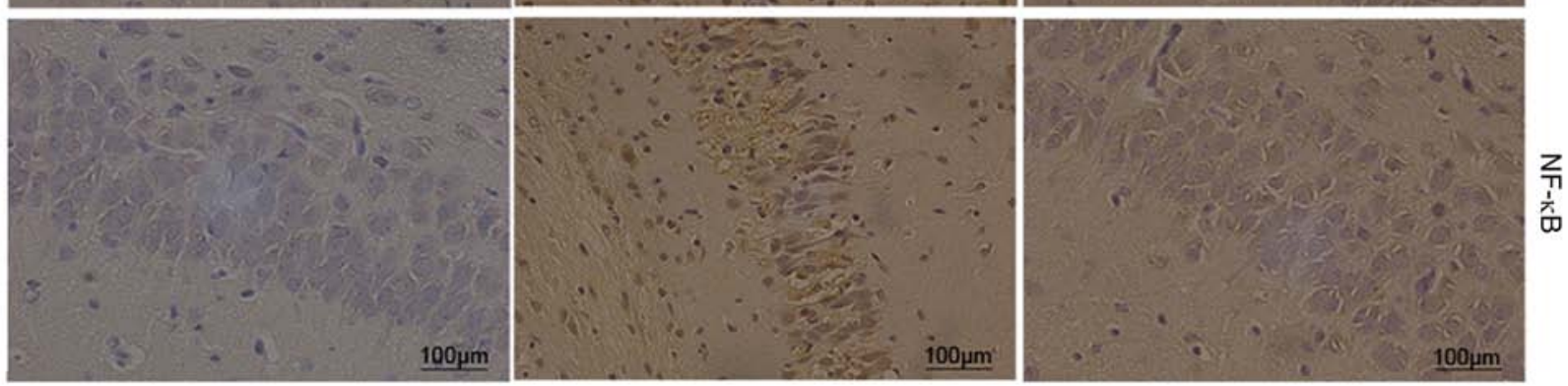

$\mathrm{D}$

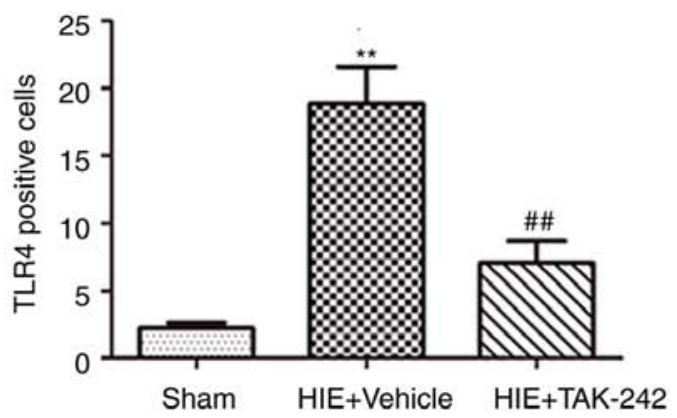

E

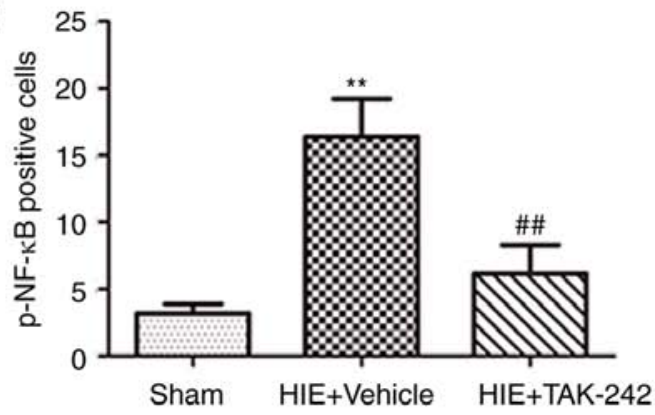

Figure 3. TAK-242 alleviates the pathological damage in brain tissues after HIE, and decreases the expression levels of TLR4 and p-NF- $\mathrm{BB}$ immunohistochemical positive cells. (A) Representative hematoxylin and eosin staining of the left hippocampus in each group. Representative (B) TLR4 and (C) p-NF- $\kappa B$ immunohistochemistry staining of the left hippocampus in each group. Expression levels of (D) TLR4 and (E) p-NF-kB positive cells in the hippocampus were significantly increased $48 \mathrm{~h}$ after HIE, while $3 \mathrm{mg} / \mathrm{kg}$ TAK-242 significantly decreased the number of positive cells. Magnification, $\mathrm{x} 400$. Scale bar, $100 \mu \mathrm{m}, \mathrm{n}=6$ per group. ${ }^{* *} \mathrm{P}<0.01$ vs. sham group; ${ }^{\# \#} \mathrm{P}<0.01$ vs. HIE + vehicle group. HIE, hypoxic-ischemic encephalopathy; TLR4, Toll like receptor 4 ; p-, phosphorylated.

Therefore, TAK-242 may exert a neuroprotective effect after HIE by inhibiting the TLR4/MyD88/TRIF/NF-kB signaling pathway and reducing the release of inflammatory cytokines.

HIE is a hypoxic-ischemic brain damage caused by perinatal asphyxia (1). Inflammatory and immune responses serve an essential role in brain ischemic injury (32). Therefore, inhibition of secondary brain injury caused by inflammatory and immune responses after hypoxia-ischemia may be a novel target for treating HIE $(1,10)$. TLR4 can regulate the process of signal transduction in many immune and inflammatory diseases, and is crucial in the inflammatory and immune responses associated with the central nervous system (33).
Activation of the TLR4/NF- $\mathrm{BB}$ signaling pathway and the release of inflammatory cytokines are present in the process of brain damage caused by carbon monoxide poisoning, trauma and cerebral hemorrhage, and inhibition of the TLR4/NF- $\mathrm{KB}$ signaling pathway reduces the degree of brain damage $(17,19,20,28)$. Furthermore, activation of the TLR4/NF- $\mathrm{KB}$ signaling pathway is also involved in cerebral ischemia-reperfusion injury in rats, and inhibition of this signaling pathway can alleviate cerebral ischemia-reperfusion injury $(16,18)$. In the present study, significant damage occurred to the neurons in brain tissue, along with deficits in neurobehavioral function in neonatal rats after HIE. In addition, large cerebral infarction appeared in the left brain 

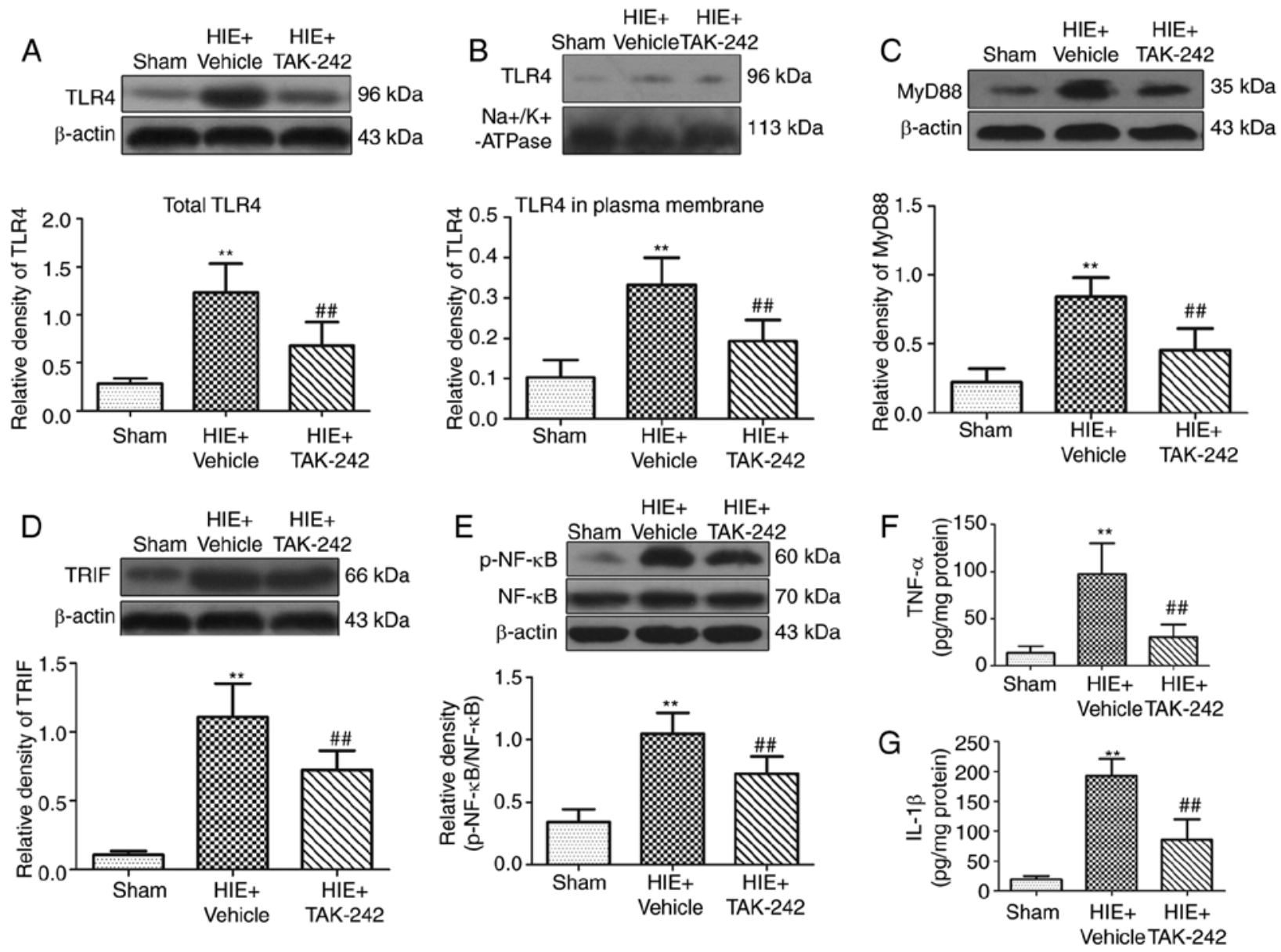

Figure 4. TAK-242 inhibits protein expression levels of the TLR4/MyD88/TRIF/NF- $\mathrm{KB}$ signaling pathway and the release of downstream inflammatory cytokines in the hippocampus. Western blotting results of TLR4, MyD88, TRIF and p-NF- $\mathrm{kB}$ in each group demonstrated that $3 \mathrm{mg} / \mathrm{kg}$ TAK-242 significantly inhibited the protein expression levels of total (A) TLR4, (B) TLR4 in plasma membrane, (C) MyD88, (D) TRIF and (E) p-NF- $\mathrm{kB}$ in the hippocampus $48 \mathrm{~h}$ after HIE. ELISA results suggested that $3 \mathrm{mg} / \mathrm{kg}$ TAK-242 significantly decreased the levels of (F) TNF- $\alpha$ and (G) IL-1 $\beta$ in the hippocampus $48 \mathrm{~h}$ after HIE. $\mathrm{n}=6 \mathrm{per}$ group. ${ }^{* *} \mathrm{P}<0.01$ vs. sham group; ${ }^{\# / P} \mathrm{P}<0.01$ vs. HIE + vehicle group. HIE, hypoxic-ischemic encephalopathy; TLR4, Toll like receptor 4; p-, phosphorylated; TRIF, TIR-domain-containing adapter-inducing interferon- $\beta$; IL, interleukin.

and cerebral edema content was also significantly increased. The present study also demonstrated that the expression levels of TLR4, p-NF- $\kappa$ B, TNF- $\alpha$ and IL-1 $\beta$ were significantly increased in the left hippocampus, indicating that the activation of TLR4/NF- $\mathrm{KB}$ signaling pathway and release of inflammatory cytokines occurred during brain damage after HIE. The present results were in line with results from Yao et al (34). However, Yao et al (34) did not elucidate the specific molecular signaling mechanisms regarding the activation of TLR4 and its downstream inflammatory cytokines after hypoxia.

TLR4 activates innate immune cells and inflammatory responsecells, and causes the release of theinflammatorycytokines TNF- $\alpha$, IL-1 $\beta$, IL-6 and IL-8 via MyD88- and TRIF-dependent signaling pathways, eventually leading to the activation of various immuno-inflammatory responses (35). Previous studies have demonstrated that the expression levels of TLR4 and its downstream signaling molecules MyD88, TRIF and NF- $\mathrm{BB}$ are upregulated in the hippocampus after traumatic brain injury in rats (20). Therefore, TLR4 may activate NF- $\kappa \mathrm{B}$ via MyD88- and TRIF-dependent signaling pathways, and promote the release of inflammatory cytokines, such as TNF- $\alpha$ and IL- $1 \beta$, further aggravating the degree of brain damage; inhibition of the TLR4/MyD88/TRIF/NF- $\kappa$ B signaling pathway plays a neuropro- tective role after brain trauma (20,36). The activation of TLR4 and its downstream signaling molecules MyD88, TRIF and NF- $\kappa \mathrm{B}$ are observed in a mouse model with cerebral hemorrhage, and inhibition of the TLR4/MyD88/TRIF/NF- $\kappa \mathrm{B}$ signaling pathway alleviates the degree of inflammatory brain damage and impaired neurological function in mice (28). The present results suggested that the levels of TLR4, p-NF- $\kappa$ B, MyD88, TRIF, TNF- $\alpha$ and IL-1 $\beta$ were increased in the left hippocampus of neonatal rats after HIE, indicating that TLR4 may activate $\mathrm{NF}-\kappa \mathrm{B}$ to promote the release of downstream inflammatory cytokines via a signaling pathway mediated by MyD88 and TRIF. Therefore, the activation of the TLR4/MyD88/TRIF/NF- $\kappa \mathrm{B}$ signaling pathway and release of inflammatory cytokines after HIE may be important in the pathogenesis of HIE, and inhibition of this signaling pathway may be a novel target for HIE treatment.

TAK-242 is a specific TLR4 inhibitor that binds to Cys747 in the intracellular domain of TLR4 and reduces TLR4 activity (37). Previous studies have demonstrated that TAK-242 plays a neuroprotective role by inhibiting the TLR4 signaling pathway, and reducing the activation and release of inflammatory cytokines $(16,20,38,39)$. At present, no studies are available on the effect of TAK-242 on HIE. Therefore, in the present study, intraperitoneal injection 
of TAK-242 $(25,27,28)$ results in a significant reduction of neurobehavioral functional deficits in neonatal HIE rats, and the pathological morphology of brain tissue was improved. Moreover, the cerebral edema content and infarct volume were significantly reduced, and the levels of TLR4, MyD88, TRIF, $\mathrm{p}-\mathrm{NF}-\kappa \mathrm{B}, \mathrm{TNF}-\alpha$ and IL-1 $\beta$ in hippocampus were also significantly reduced. The present results suggested that TAK-242 may alleviate brain damage caused by hypoxia-ischemia by inhibiting the TLR4/MyD88/TRIF/NF- $\mathrm{BB}$ signaling pathway and reducing the release of inflammatory cytokines, thereby exerting a neuroprotective effect after HIE.

In conclusion, to the best of our knowledge, the present study was the first to use the neonatal HIE rat model to identify that TAK-242 may serve a neuroprotective role in the brain tissues after HIE. This neuroprotective effect is suggested to be via an inhibition of the TLR4/MyD88/TRIF/NF- $\kappa \mathrm{B}$ signaling pathway and a reduction in the release of inflammatory cytokines. Therefore, TAK-242 may be a promising medication for HIE. However, due to the limited sample size and the fact that not all pathological features of HIE were included in the present study, further studies are required to investigate the neuroprotective function of TAK-242 in HIE.

\section{Acknowledgements}

Not applicable.

\section{Funding}

This study was supported by the National Natural Science Foundation of China (grant no. 81771625).

\section{Availability of data and materials}

All data generated or analyzed during this study are included in this published article.

\section{Authors' contributions}

LJ and XF conceived and designed the experiments. LJ, ZX, HL, MW, FW, SL and JT performed the experiments and analyzed the data. LJ and ZX wrote the manuscript. All authors read and approved the manuscript and agree to be accountable for all aspects of the research in ensuring that the accuracy or integrity of any part of the work are appropriately investigated and resolved.

\section{Ethics approval and consent to participate}

All animal experimental procedures were approved by the Laboratory Animal Ethics Committee of Yangzhou University [approval no. SCXK (Su) 2017-0007], and were conducted in strict accordance with the Regulation on the Administration of Laboratory Animals issued by the Ministry of Science and Technology of the People's Republic of China. All efforts were made to reduce the suffering of animals.

\section{Patient consent for publication}

Not applicable.

\section{Competing interests}

The authors declare that they have no competing interests.

\section{References}

1. Qin X, Cheng J, Zhong Y, Mahgoub OK, Akter F, Fan Y, Aldughaim M, Xie Q, Qin L, Gu L, et al: Mechanism and treatment related to oxidative stress in neonatal hypoxic-ischemic encephalopathy. Front Mol Neurosci 12: 88, 2019.

2. Zhao M, Zhu P, Fujino M, Zhuang J, Guo H, Sheikh I, Zhao L and Li XK: Oxidative stress in hypoxic-ischemic encephalopathy: Molecular mechanisms and therapeutic strategies. Int J Mol Sci 17: E2078, 2016.

3. Liu L, Oza S, Hogan D, Perin J, Rudan I, Lawn JE, Cousens S, Mathers $\mathrm{C}$ and Black RE: Global, regional, and national causes of child mortality in 2000-13, with projections to inform post-2015 priorities: An updated systematic analysis. Lancet 385: 430-440, 2015.

4. Gale C, Statnikov Y, Jawad S, Uthaya SN and Modi N; Brain Injuries expert working group: Neonatal brain injuries in England: Population-based incidence derived from routinely recorded clinical data held in the national neonatal research database. Arch Dis Child Fetal Neonatal Ed 103: F301-F306, 2018.

5. Leigh S, Granby P, Turner M, Wieteska S, Haycox A and Collins B: The incidence and implications of cerebral palsy following potentially avoidable obstetric complications: A preliminary burden of disease study. BJOG 121: 1720-1728, 2014.

6. Smith AL, Alexander M, Rosenkrantz TS, Sadek ML and Fitch RH: Sex differences in behavioral outcome following neonatal hypoxia ischemia: Insights from a clinical meta-analysis and a rodent model of induced hypoxic ischemic brain injury. Exp Neurol 254: 54-67, 2014.

7. Doycheva D, Shih G, Chen H, Applegate R, Zhang JH and Tang J: Granulocyte-colony stimulating factor in combination with stem cell factor confers greater neuroprotection after hypoxic-ischemic brain damage in the neonatal rats than a solitary treatment. Transl Stroke Res 4: 171-178, 2013.

8. Shetty J: Neonatal seizures in hypoxic-ischaemic encephalopathy-risks and benefits of anticonvulsant therapy. Dev Med Child Neurol 57 (Suppl 3): S40-S43, 2015.

9. Knox R, Brennan-Minnella AM, Lu F, Yang D, Nakazawa T, Yamamoto T, Swanson RA, Ferriero DM and Jiang X: NR2B phosphorylation at tyrosine 1472 contributes to brain injury in a rodent model of neonatal hypoxia-ischemia. Stroke 45: 3040-3047, 2014.

10. Jenkins DD, Rollins LG, Perkel JK, Wagner CL, Katikaneni LP, Bass WT, Kaufman DA, Horgan MJ, Languani S, Givelichian L, et al: Serum cytokines in a clinical trial of hypothermia for neonatal hypoxic-ischemic encephalopathy. J Cereb Blood Flow Metab 32: 1888-1896, 2012.

11. Leitner GR, Wenzel TJ, Marshall N, Gates EJ and Klegeris A: Targeting toll-like recepter 4 to modulate neuroinflammation in central nervous system disorders. Expert Opin Ther Targets 23: 865-882, 2019.

12. Tajalli-Nezhad S, Karimian M, Beyer C, Atlasi MA and Azami Tameh A: The regulatory role of Toll-like receptors after ischemic stroke: Neurosteroids as TLR modulators with the focus on TLR2/4. Cell Mol Life Sci 76: 523-537, 2019.

13. Mulero MC, Huxford T and Ghosh G: NF-kB, IkB, and IKK: Integral components of immune system signaling. Adv Exp Med Biol 1172: 207-226, 2019.

14. Brown J, Wang H, Hajishengallis $\mathrm{GN}$ and Martin M: TLR-signaling networks: An integration of adaptor molecules, kinases, and cross-talk. J Dent Res 90: 417-427, 2011.

15. Liu Y and Fassbender K: Deficiency of TLR4 ameliorates hypoperfusion-induced brain pathology. Theranostics 8: 6355-6356, 2018.

16. Zhao H, Chen Z, Xie LJ and Liu GF: Suppression of TLR4/NF-kB signaling pathway improves cerebral ischemia-reperfusion injury in rats. Mol Neurobiol 55: 4311-4319, 2018.

17. Pang L, Zhang N, Dong N, Wang DW, Xu DH, Zhang $\mathrm{P}$ and Meng XW: Erythropoietin protects rat brain injury from carbon monoxide poisoning by inhibiting toll-like receptor 4/NF-kappa B-dependent inflammatory responses. Inflammation 39: 561-568, 2016.

18. Wang Y, Ge P, Yang L, Wu C, Zha H, Luo T and Zhu Y: Protection of ischemic post conditioning against transient focal ischemia-induced brain damage is associated with inhibition of neuroinflammation via modulation of TLR 2 and TLR4 pathways. J Neuroinflammation 11: 15, 2014. 
19. Feng Y, Cui C, Liu X, Wu Q, Hu F, Zhang H, Ma Z and Wang L: Protective role of apocynin via suppression of neuronal autophagy and TLR4/NF-kB signaling pathway in a rat model of traumatic brain injury. Neurochem Res 42: 3296-3309, 2017.

20. Feng Y, Gao J, Cui Y, Li M, Li R, Cui C and Cui J: Neuroprotective effects of resatorvid against traumatic brain injury in rat: Involvement of neuronal autophagy and TLR4 signaling pathway. Cell Mol Neurobiol 37: 155-168, 2017.

21. Liu T, Liu M, Zhang T, Liu W, Xu H, Mu F, Ren D, Jia N, Li Z, Ding Y, et al: Z-Guggulsterone attenuates astrocytes-mediated neuroinflammation after ischemia by inhibiting toll-like receptor 4 pathway. J Neurochem 147: 803-815, 2018.

22. Liu FY, Cai J, Wang C, Ruan W, Guan GP, Pan HZ, Li JR, Qian C, Chen JS, Wang L and Chen G: Fluoxetine attenuates neuroinflammation in early brain injury after subarachnoid hemorrhage: A possible role for the regulation of TLR4/MyD88/NF-KB signaling pathway. J Neuroinflammation 15: 347, 2018.

23. Tang R, Lin YM, Liu HX and Wang ES: Neuroprotective effect of docosahexaenoic acid in rat traumatic brain injury model via regulation of TLR4/NF-Kappa B signaling pathway. Int J Biochem Cell Biol 99: 64-71, 2018.

24. Hussey SE, Liang H, Costford SR, Klip A, DeFronzo RA, Sanchez-Avila A, Ely B and Musi N: TAK-242, a small-molecule inhibitor of Toll-like receptor 4 signalling, unveils similarities and differences in lipopolysaccharide- and lipid-induced inflammation and insulin resistance in muscle cells. Biosci Rep 33: 37-47, 2012.

25. Hua F, Tang H, Wang J, Prunty MC, Hua X, Sayeed I and Stein DG: TAK-242, an antagonist for Toll-like receptor 4 protects against acute cerebral ischemia/reperfusion injury in mice. J Cereb Blood Flow Metab 35: 536-542, 2015.

26. Vannucci RC, Connor JR, Mauger DT, Palmer C, Smith MB Towfighi J and Vannucci SJ: Rat model of perinatal hypoxic-ischemic brain damage. J Neurosci Res 55: 158-163, 1999.

27. Hwang JW, Jeon YT, Lim YJ and Park HP: Sevoflurane postconditioning-induced anti-inflammation via inhibition of the toll-like receptor-4/nuclear factor Kappa B pathway contributes to neuroprotection against transient global cerebral ischemia in rats. Int J Mol Sci 18: E2347, 2017.

28. Wang YC, Wang PF, Fang H, Chen J, Xiong XY and Yang QW: Toll-like receptor 4 antagonist attenuates intracerebra hemorrhage-induced brain injury. Stroke 44: 2545-2552, 2013.

29. Longa EZ, Weinstein PR, Carlson S and Cummins R: Reversible middle cerebral artery occlusion without craniectomy in rats. Stroke 20: 84-91, 1989.
30. Li Z, Wang J, Zhao C, Ren K, Xia Z, Yu H and Jiang K: Acute blockage of notch signaling by DAPT induces neuroprotection and neurogenesis in the neonatal rat brain after stroke. Transl Stroke Res 7: 132-140, 2016.

31. Cui C, Cui Y, Gao J, Sun L, Wang Y, Wang K, Li R, Tian Y, Song S and Cui J: Neuroprotective effect of ceftriaxone in a rat model of traumatic brain injury. Neurol Sci 35: 695-700, 2014.

32. Ystgaard MB, Scheffler K, Suganthan R, Bjoras M, Ranheim T, Sagen EL, Halvorsen B, Saugstad OD and Yndestad A: Neuromodulatory effect of NLRP3 and ASC in neonatal hypoxic ischemic encephalopathy. Neonatology 115: 355-362, 2019.

33. Kong Y and Le Y: Toll-like receptors in inflammation of the central nervous system. Int Immunopharmacol 11: 1407-1414, 2011.

34. Yao L, Kan EM, Lu J, Hao A, Dheen ST, Kaur C and Ling EA: Toll-like receptor 4 mediates microglial activation and production of inflammatory mediators in neonatal rat brain following hypoxia: Role of TLR4 in hypoxic microglia. J Neuroinflammation 10: 23, 2013.

35. Li M, Liu J, Bi Y, Chen J and Zhao L: Potential medications or compounds acting on toll-like receptors in cerebral ischemia. Curr Neuropharmacol 16: 160-175, 2018.

36. Zhu HT, Bian C, Yuan JC, Chu WH, Xiang X, Chen F, Wang CS, Feng $\mathrm{H}$ and Lin JK: Curcumin attenuates acute inflammatory injury by inhibiting the TLR4/MyD88/NF-KB signaling pathway in experimental traumatic brain injury. $\mathrm{J}$ Neuroinflammation 11: $59,2014$.

37. Lei C, Wu B, Cao T, Liu M and Hao Z: Brain recovery mediated by toll-like receptor 4 in rats after intracerebral hemorrhage. Brain Res 1632: 1-8, 2016

38. Zhao Y, Zhao Y, Zhang M, Zhao J, Ma X, Huang T, Pang H, Li J and Song J: Inhibition of TLR4 signalling-induced inflammation attenuates secondary injury after diffuse axonal injury in rats. Mediators Inflamm 2016: 4706915, 2016.

39. Li XQ, Lv HW, Tan WF, Fang B, Wang H and Ma H: Role of the TLR4 pathway in blood-spinal cord barrier dysfunction during the bimodal stage after ischemia/reperfusion injury in rats. J Neuroinflammation 11: 62, 2014.

This work is licensed under a Creative Commons Attribution-NonCommercial-NoDerivatives 4.0 International (CC BY-NC-ND 4.0) License. 